\title{
Ictal asystole: a diagnostic and management conundrum
}

\author{
Gashirai K Mbizvo ${ }^{1,2}$, Chris Derry ${ }^{3,4}$, Richard Davenport ${ }^{5}$
}

Appendix A: Ictal asystole - the patient perspective

This information was kindly provided to us by patient two from the case report.

\section{Signalling question}

What has been your overall experience with this condition?

\section{Patient's response}

"My first seizure was a shock but in hindsight not a surprise as I had been having episodes of 'déjà vu' for several years prior. I had collapsed in 2009 and underwent several tests both for neuro and cardiac causes. No abnormalities were detected at that time.

After my seizure, I was admitted to hospital overnight and then went for tests to look for what may have caused the seizure. I was started on antiepileptic medication and was told I had epilepsy. I stopped driving but carried on working. I had some other tests including a heart trace monitor for two days. When I was wearing the monitor, I passed out again. When I handed the heart trace back to the clinic later that day, one of the doctors called and explained that my heart rhythm was too slow during the symptoms and that I needed a pacemaker. I went in and had the procedure the next day.

The doctors now thought it was the abnormal heart rhythm that caused the seizure and not epilepsy, so they took me off the medication and told me I could reapply for my driving licence. But six weeks later, I had another seizure even though I had a pacemaker; so unfortunately the problem has been epilepsy and an abnormal heart rate."

How did it make you feel when you "My first thought was about not being able to drive and losing my independence. I were first told you had a diagnosis of epilepsy? was also very aware that my seizures happened in front of colleagues and loved ones so I felt very vulnerable and the thought of losing control is frightening even now."

How did it then make you feel when you "I felt fantastic!! I could reapply for my licence and I was 'fixed'. Ok - I had a were then told you didn't have epilepsy? pacemaker but that gave me a sense of security that it would trigger if my heart went too slow. So no more seizures - I felt relieved by that."

How did it make you feel when you had "I was extremely disappointed and frustrated as I had been given two diagnoses a seizure whilst off medication thinking that you didn't have epilepsy?

- then one was excluded but then it reappeared with no warning. I was also upset for my husband as he witnessed the second seizure and that might not have happened if I had kept taking the medication."

What has been the impact of having a "Epilepsy has not really changed my life very much (....except for the driving diagnosis of epilepsy on your life? What part) but I'm used to coping with medical conditions so I have learned to would you like to see done better for others who may have a similar diagnosis to yours? 'just get on with it!!' My hope is that the medication continues to control the seizures so I don't have any more. In future, I would hope to see more collective decisions made about diagnosis and management for people with more than one symptom or diagnosis."

${ }^{1} \mathrm{PhD}$ Clinical Research Fellow, Muir Maxwell Epilepsy Centre, Centre for Clinical Brain Sciences, The University of Edinburgh, Edinburgh, UK; ${ }^{2} \mathrm{PhD}$ Clinical Research Fellow, Department of Clinical Neurosciences, Western General Hospital, Edinburgh, UK; ${ }^{3,5}$ Consultant Neurologist, Department of Clinical Neurosciences, Western General Hospital, Edinburgh, UK; ${ }^{4}$ Honorary Clinical Senior Lecturer, NHS Research Fellow, Department of Clinical Neurosciences and Sleep Medicine, Centre for Clinical Brain Sciences, The University of Edinburgh, Edinburgh, UK 